\title{
Near-field analysis of surface electromagnetic waves in the bandgap region of a polymeric grating written on a one-dimensional photonic crystal
}

\author{
Tristan Sfez, ${ }^{1, a)}$ Emiliano Descrovi, ${ }^{2}$ Lorenzo Dominici, ${ }^{3, c}$ Wataru Nakagawa, ${ }^{1, b)}$ \\ Francesco Michelotti, ${ }^{3}$ Fabrizio Giorgis, ${ }^{2}$ and Hans-Peter Herzig ${ }^{1}$ \\ ${ }^{1}$ Institut de Microtechnique, Université de Neuchâtel, rue A.-L. Breguet 2, 2000 Neuchâtel, Switzerland \\ ${ }^{2}$ Dipartimento di Fisica, Politecnico di Torino, corso Duca degli Abruzzi 24, 10129 Torino, Italy \\ ${ }^{3}$ Dipartimento di Energetica, LA SAPIENZA Università di Roma, via A. Scarpa 16, 00161 Roma, Italy
}

(Received 14 May 2008; accepted 24 July 2008; published online 14 August 2008)

\begin{abstract}
The spatial distribution of surface electromagnetic waves on a one-dimensional photonic crystal with a polymeric grating on top is mapped using a multiheterodyne scanning near-field optical microscope. The grating opens a bandgap. At the band edges, a stationary field formed by two surface waves with opposite propagation constants and almost identical amplitudes is produced. Field intensity maxima are localized to the grating peaks or grooves, depending on the wavelength. Far from the bandgap, we identify surface waves coupled via either the 0 or the -1 order of the grating. The corresponding phase profiles indicate that these waves propagate in opposite directions. (C) 2008 American Institute of Physics. [DOI: 10.1063/1.2970961]
\end{abstract}

Surface electromagnetic waves (SEWs) are nonradiative waves confined at the interface between two media. ${ }^{1}$ SEWs can exist provided that the dielectric functions of the two surrounding media satisfy the relationship $\varepsilon_{i}(\omega) / \varepsilon_{j}(\omega)<-1$. Such a condition can be attained at the physical boundaries of artificial structures such as photonic crystals (PCs). ${ }^{2}$ SEWs on truncated periodic structures are also called Bloch surface waves or Tamm states. In the literature, extensive work on SEWs at optical and near-infrared (NIR) frequencies can be found (see, e.g., Refs. 3-5 and references therein).

In this letter, we consider SEWs on a dielectric structure constituted by a silicon nitride one-dimensional PC (1DPC) with a subwavelength dielectric grating on the top surface. There is a growing interest in investigating the interaction of surface waves with periodic structures. Typically, gratings on multilayers are used for mode coupling. ${ }^{6}$ Nevertheless, it has been recently suggested that shallow, periodic corrugations realized on top of $1 \mathrm{DPC}$ can be used in biosensing applications. ${ }^{7}$ For well defined grating geometries, the corrugation opens a bandgap in the dispersion relation of the surface mode. ${ }^{8}$ Such a bandgap can be fruitfully used for enhancing the amplitude of the surface wave, ${ }^{9}$ for increasing the density of electromagnetic modes,${ }^{10}$ or for guiding. ${ }^{11}$ In this framework, a near-field microscopy analysis can give important insights on the spatial distribution of complex optical field close to structured surfaces. In the following, we provide a direct visualization of the surface wave propagation as affected by the presence of the grating.

In a recent paper, ${ }^{12}$ the authors demonstrated that an energy bandgap for SEWs is obtained by means of a polymeric subwavelength grating on a 1DPC. The structure is shown in Fig. 1(a). The silicon nitride multilayer, grown by plasma enhanced chemical vapor deposition, is composed of a ten-

\footnotetext{
a)Electronic mail: tristan.sfez@unine.ch.

${ }^{b)}$ Present address: Electrical and Computer Engineering, Montana State University, P.O. Box 173780 Bozeman, MT 59717-3780, USA.

${ }^{c)}$ Present address: CHOSE, Dept. of Electronics Eng., "Tor Vergata" University, Via del Politecnico 1, 00133 Roma, Italy.
}

period stack of high $\left(n_{H}=2.23\right.$ at $\left.\lambda=1530 \mathrm{~nm}\right)$ and low $\left(n_{L}\right.$ $=1.75$ at $\lambda=1530 \mathrm{~nm})$ refractive index layers having thickness $d_{H}=240 \mathrm{~nm}$ and $d_{L}=294 \mathrm{~nm}$, respectively. This $1 \mathrm{DPC}$ sustains SEWs in the NIR region, as recently demonstrated in Ref. 13. A $110 \mathrm{~nm}$ thick asobenzene side-chain copolymer film (DR1-PMMA, $n_{p}=1.67$ ) spin coated on the air-1DPC interface is holographically illuminated to produce a shallow grating whose period and amplitude are $\Lambda=610 \mathrm{~nm}$ and $h$ $=55 \mathrm{~nm}$, respectively. We couple SEWs in the Kretschmann configuration by means of a BK7-glass prism. A multiheterodyne scanning near-field optical microscope (SNOM) (described in detail in Ref. 13) is used to locally collect the near-field by raster scanning the corrugated surface of the sample with a dielectric probe (Lovalite, $70 \mathrm{~nm} \mathrm{Al}$ coated, aperture $200 \mathrm{~nm}$ ). Surface topography is obtained by means of shear-force detection. This original SNOM setup allows simultaneous measurements of the amplitude and phase of optical near-fields resulting from two orthogonal electric field components of the illuminating beam. In our experimental geometry, we simultaneously illuminate the sample with a superposition of linearly TE and TM polarized beams. The TE-polarized light couples to the SEWs. The incident light is shaped with either a snap-on collimator or a focuser
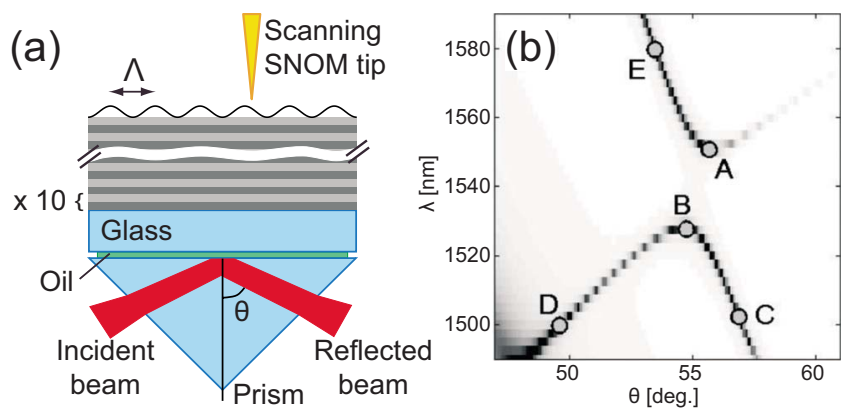

FIG. 1. (Color online) (a) Diagram of the 1DPC with the grating on top SEW are coupled in the Kretschmann configuration with TE-polarized illumination. (b) Calculated reflectance map $R(\theta, \lambda)$ of the corrugated 1DPC Illumination is TE-polarized. Darker regions represent low reflectance. The numbered circles indicate the points $(\theta, \lambda)$ considered in the SNOM analysis. 

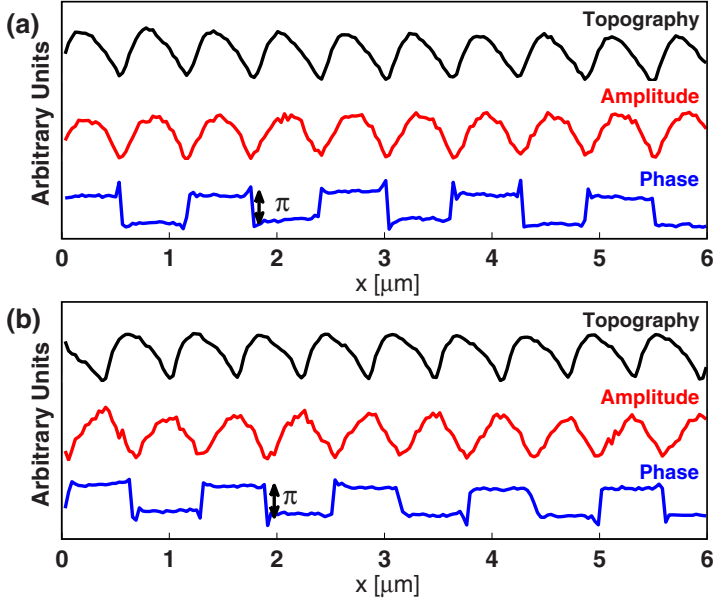

FIG. 2. (Color online) Cross-sectional profiles of topography, amplitude, and phase at (a) the low-frequency (point A) and (b) high-frequency (point B) band edges. The amplitude and phase are produced by TE-polarized illumination beam.

mounted on a goniometer. The grating grooves are placed perpendicular to the plane of incidence. We use a charge coupled device camera to collect the reflected light and check SEW coupling in a classical $M$-line configuration.

Calculations performed with the well known $C$-method ${ }^{14}$ show that the grating opens a bandgap in the SEW dispersion curve [Fig. 1(b)], as experimentally measured in Ref. 12. The black lines identify low-reflectance regions and are associated with SEW coupling. In the following, we focus on five significant points marked by circles on the SEW dispersion curve. We consider the near-field distribution at the band edges (point $\mathrm{A}$ at $\lambda_{A}=1547 \mathrm{~nm}, \theta_{A}=55.3^{\circ}$ and point $\mathrm{B}$ at $\lambda_{B}=1524 \mathrm{~nm}, \theta_{B}=54.5^{\circ}$ ) and outside the bandgap (point $\mathrm{C}$ at $\lambda_{C}=1502 \mathrm{~nm}, \theta_{C}=56.9^{\circ}$, point $\mathrm{D}$ at $\lambda_{D}=1500 \mathrm{~nm}, \theta_{D}$ $=49.6^{\circ}$ and point $\mathrm{E}$ at $\lambda_{E}=1580 \mathrm{~nm}, \theta_{E}=53.5^{\circ}$ ).

In order to maximize the SEW coupling strength, a collimated beam of about $500 \mu \mathrm{m}$ is used for illumination. Once SEW coupling is obtained, linear scans of $6 \mu \mathrm{m}$ on the corrugated 1DPC surface are performed. Points A and B are considered first. In Fig. 2, we show the measurements at the low-frequency (A) and high-frequency (B) band edges, respectively. Although the sinusoidal topographic profile of the grating appears deformed because of the convolution with the SNOM tip, ${ }^{15}$ these peaks and grooves are well defined.

The measured near fields show an amplitude distribution having the same periodicity as the grating. Similarly to planar Bragg reflectors, the high-energy and low-energy fields have intensity maxima localized into the low- $\varepsilon$ regions (air band) and high- $\varepsilon$ regions (dielectric band) respectively. ${ }^{8}$ In both cases, the phase shows a squared profile with a $\pi$-shift occurring at amplitude minima. This is a typical effect of interference between two counterpropagating waves having equal amplitudes, i.e., the modes at the band edges are standing waves.

Outside the bandgap region, we focus our attention to points C-E. Results are shown in Fig. 3. Beside the opening of a bandgap, the grating produces a branch folding of the SEW dispersion curve ${ }^{12}$ that is particularly evident at wavelengths shorter than $\lambda=1524 \mathrm{~nm}$ (point B). The near-field amplitudes associated with points $\mathrm{C}$ and $\mathrm{D}$ show a constant spatial distribution with very slight modulations almost comparable with the noise level of the whole detection process.
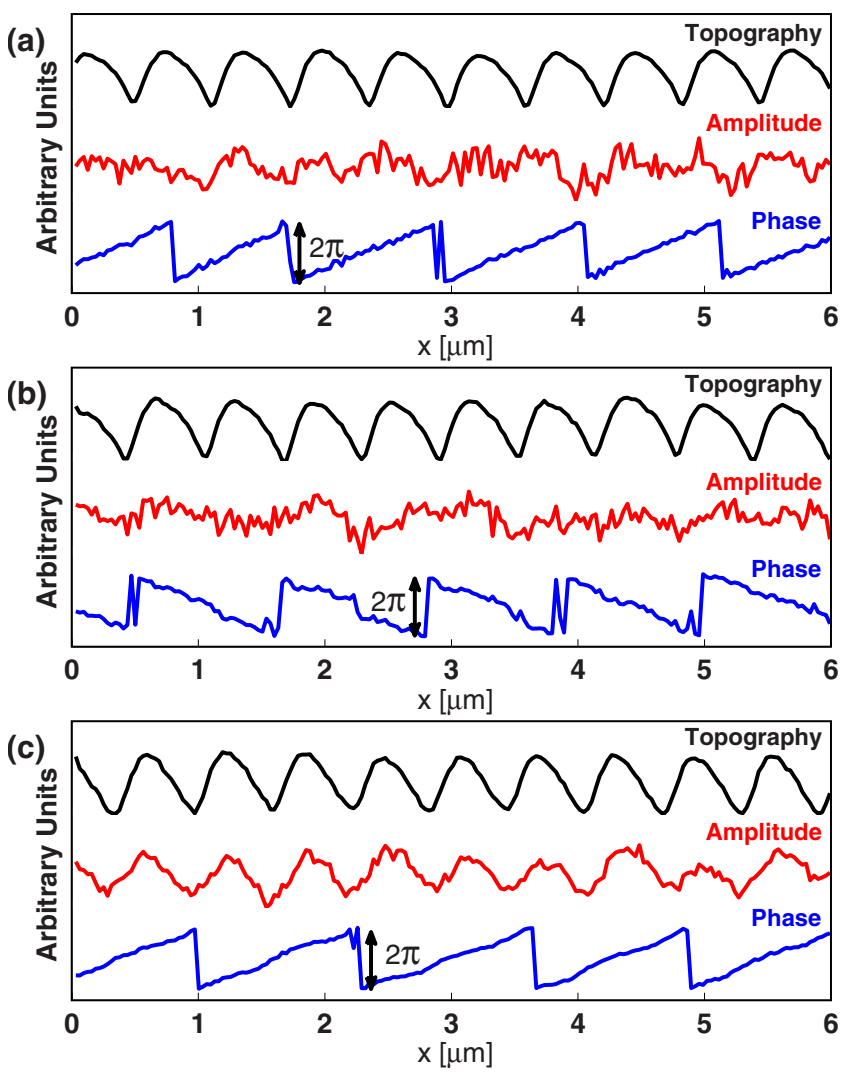

FIG. 3. (Color online) Cross-sectional profiles of topography, amplitude, and phase at optical frequencies and incident angles corresponding to (a) point $\mathrm{C}$, (b) point $\mathrm{D}$, and (c) point E. As shown in Fig. 1(b), the amplitude and phase are associated to an optical near-field produced by a TE-polarized illumination beam.

The phase distributions exhibit the typical saw-tooth profile associated with propagating waves with a well-defined propagation constant. The periodicity of the phases is almost the same, but the SEW travel in opposite directions. In particular, the SEW at point $C$ is characterized by a theoretically calculated positive propagation constant $\beta_{C}$ $=2 \pi n_{B K 7} / \lambda \cdot \sin \theta_{C}$, while the SEW at point $\mathrm{D}$ has a negative $\beta_{D}=2 \pi n_{B K 7} / \lambda \cdot \sin \theta_{D}-2 \pi / \Lambda$, being coupled through the -1 order of the grating. Within this framework, SEW lying on the right branch of the dispersion curve at frequencies higher than the bandgap are coupled to the 0 order of the grating, while SEW on the left branch are efficiently coupled via the -1 order. At the band edges, the two branches merge and the counterpropagating waves are coupled simultaneously, resulting in the interference pattern shown in Fig. 2.

At frequencies lower than the bandgap, calculations show that only one branch of the dispersion curve is clearly visible. The SEW coupling efficiency via the -1 order of the grating is very small. At point $\mathrm{E}$, we again detect a saw-tooth phase profile indicating a positive propagation constant. A slight modulation of the near-field amplitude is also found, as expected from rigorous calculations. The two counterpropagating SEW that are simultaneously coupled at band edges can be imaged in the direct space by using a weakly focused illumination beam of about $20 \mu \mathrm{m}$. We illuminate at $\left(\lambda_{A}, \theta_{A}\right)$ and $\left(\lambda_{B}, \theta_{B}\right)$ corresponding to the points $\mathrm{A}$ and $\mathrm{B}$ in Fig. 1(b) and detect both TE- and TM-polarized beams. Near-field measurements are performed over a scan region of 90 $\times 90 \mu \mathrm{m}^{2}$, with a linear sampling interval of $\Delta x=1.4 \mu \mathrm{m}$. Since the measured field is modulated with period $\Lambda$ 


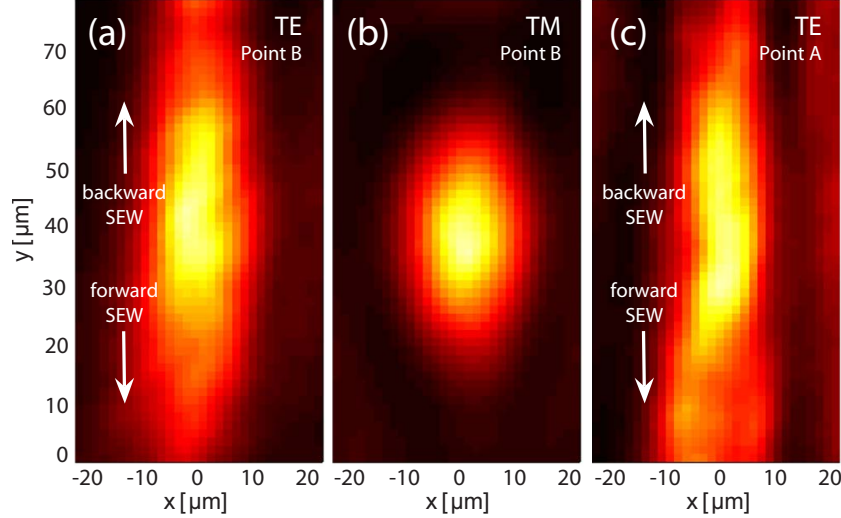

FIG. 4. (Color online) Normalized intensity distribution of the near-field obtained at the band edges. (a) TE and (b) TM-polarized incident beam at point B, (c) TE at point A. The illumination beam provides a spot of size $20 \mu \mathrm{m}$.

$=0.610 \mu \mathrm{m}$, we expect an aliasing effect. After proper Fourier filtering, we obtained the near-field intensity maps presented in Fig. 4. The TE-polarized near-field distributions show a strongly elongated distribution parallel to the illumination direction as compared to the TM-polarized field. This difference indicates SEW coupling. Nevertheless, while in the case of SEW coupling on flat multilayers the field is distributed in an asymmetric, cometlike pattern, ${ }^{13}$ in the case of SEW coupling at the band edges of a shallow grating, the distribution appears rather symmetric. This effect is due to the coupling of both forward and backward propagating SEW, traveling away from the central coupling region in opposite directions.

In this work, we presented a near-field analysis of TEpolarized surface waves coupled on a periodically corrugated 1DPC. We demonstrated that a standing SEW defined by two counterpropagating modes having almost identical ampli- tudes is generated at the band edges of the grating. The field is mostly localized at the grating grooves and peaks at the high- and low-frequency photonic band edges, respectively. Away from the bandgap, the two counterpropagating SEW can be selectively coupled via either the 0 or the -1 diffraction order of the grating. We also show that the SNOM technique can be usefully exploited to accurately characterize the optical response of subwavelength, shallow gratings when illuminated by surface waves.

This work is supported by Swiss National Science Foundation and the Centre of Excellence funded by MIUR (Italian Ministry for Education, University and Research) LATEMAR (Grant No. FIRB 2003-2004).

${ }^{1}$ A. D. Boardman, Electromagnetic Surface Modes (Wiley, Toronto, 1982).

${ }^{2}$ W. M. Robertson, J. Lightwave Technol. 17, 2013 (1999).

${ }^{3}$ P. Yeh, A. Yariv, and A. Y. Cho, Appl. Phys. Lett. 32, 104 (1978).

${ }^{4}$ W. M. Robertson, G. Arjavalingam, R. D. Meade, K. D. Brommer, A. M. Rappe, and J. D. Joannopoulos, Opt. Lett. 18, 528 (1993).

${ }^{5}$ A. S. Ramirez-Duverger, J. Gaspar-Armenta, and R. Garcia-Llamas, Opt. Commun. 277, 302 (2007).

${ }^{6}$ P. L. Rochon and L. Levesque, Opt. Express 14, 13050 (2006).

${ }^{7}$ M. Liscidini and J. E. Sipe, Appl. Phys. Lett. 91, 253125 (2007).

${ }^{8}$ W. L. Barnes, T. W. Preist, S. C. Kitson, and J. R. Sambles, Phys. Rev. B 54, 6227 (1996).

${ }^{9}$ C. J. Alleyne, A. G. Kirk, R. C. McPhedran, N. A. P. Nicorovici, and D. Maystre, Opt. Express 15, 8163 (2007).

${ }^{10}$ A. J. Benahmed and C. M. Ho, Appl. Opt. 46, 3369 (2007).

${ }^{11}$ J. C. Weeber, A. Bouhelier, G. C. des Francs, L. Markey, and A. Dereux, Nano Lett. 7, 1352 (2007).

${ }^{12}$ E. Descrovi, F. Giorgis, L. Dominici, and F. Michelotti, Opt. Lett. 33, 243 (2008).

${ }^{13}$ E. Descrovi, T. Sfez, L. Dominici, W. Nakagawa, F. Michelotti, F. Giorgis, and H.-P. Herzig, Opt. Express 16, 5453 (2008).

${ }^{14}$ L. Li, G. Granet, J. P. Plumey, and J. Chandezon, J. Opt. A, Pure Appl. Opt. 5, 141 (1996)

${ }^{15}$ E. Meyer, H. J. Hug, and R. Bennewitz, Scanning Probe Microscopy: The Lab on a Tip (Springer, Berlin, 2004). 